\title{
A Non-Data-Aided and Non-Phase-Based AFC Method for MPSK DS/CDMA Transceivers Used in Digital Broadcasting
}

\author{
Jing Lei and Tung-Sang Ng, Fellow, IEEE
}

\begin{abstract}
Due to its high-performance broadcast capability, $M$-ary phase-shift-keying (MPSK) direct sequence code division multiple access (DS/CDMA) technique is widely used in terrestrial and satellite communications to provide wired/wireless services such as audio broadcasting, video broadcasting and high-speed data transmission. However, both coherent and noncoherent MPSK modulation schemes are sensitive to the carrier frequency offset (CFO) incurred by transceiver oscillator instability and/or Doppler shift. In this paper, a nondata-aided and nonphase-based automatic frequency control (AFC) method for MPSK DS/CDMA transceivers is proposed. Different from phase-based AFC approaches, which utilize the frequency-offset-induced phase rotation and suffer from the modulo- $2 \pi$ phase ambiguity, the proposed method exploits the frequency-offset-induced power degradation and has a wide tracking range of twice the symbol rate, which is independent of the phase keying number as well as the spreading code length. To shorten the tracking time of AFC, the improved steepest descent algorithm and the reshaped $S$ curve are employed. Due to its wide tracking range, short tracking time and low implementation complexity, the proposed AFC method is attractive for terrestrial and satellite-based digital broadcasting.
\end{abstract}

Index Terms-AFC, correlator, digital broadcasting, DS/CDMA, MPSK, steepest descent methods.

\section{INTRODUCTION}

$\mathbf{M}$ OTIVATED by the growing demand for high-quality wired/wireless services such as digital audio broadcasting, digital video broadcasting and high-speed data transmission, the technique of Direct Sequence Code Division Multiple Access (DS/CDMA) has received considerable research interest all over the world [1]-[12]. $M$-ary phase-shift-keying (MPSK) has been adopted as the standard modulation scheme for DS/CDMA transceivers because of its bandwidth efficiency and implementation simplicity [13]. Due to its high-performance broadcast capability [1], [2], [4]-[8], [14], the MPSK DS/CDMA scheme has been widely used in the hub-to-mobile or satellite-to-earth-station links in communication networks such as mobile satellite systems (MSAT) and very small aperture terminal satellite systems

Manuscript received February 27, 2003; revised July 7, 2003. This work was supported by the Research Grants Council of Hong Kong Government and the University Research Committee of HKU. This paper was presented in part at the IEEE International Symposium on Circuits and System (ISCAS), Sydney, Australia, 2001.

The authors are with the Department of Electrical and Electronic Engineering, the University of Hong Kong.

Digital Object Identifier 10.1109/TBC.2003.819048
(VSAT). In addition, the MPSK DS/CDMA transceiver is also employed in the second and the third generation digital cellular standards such as IS-95, cdma2000 and WCDMA.

Nevertheless, MPSK is sensitive to the carrier frequency offset (CFO) caused by the transceiver oscillator instability and/or the Doppler shift [13], [15]. Therefore, the loop of automatic frequency control (AFC) is a necessary component in MPSK DS/CDMA receivers. In [15], Mauss, Classen and Meyr have categorized the AFC methods previously used in MPSK DS/CDMA receivers into decision-aided feedforward schemes (DAFF), nondecision-aided feedforward schemes (NDAFF) and nondecision-aided feedback schemes (NDAFB). These approaches exploit the CFO-induced phase rotation and require a large look-up table for realization. Due to the modulo- $2 \pi$ phase ambiguity, their tracking range is constrained to $(M T)^{-1}$, where $M$ and $T$ denote the modulation order and the symbol duration, respectively. This range is insufficient when CFO on the order of the symbol rate is encountered, for example, in a LEO satellite communication channel or when the AFC initially takes over from the acquisition mode. Furthermore, in order to eliminate the pattern noise resulted from data modulation, NDA schemes have to invoke computationally complex nonlinear transformations [16], while DAFF schemes rely on the decision feedback [17], [18] which, unfortunately, becomes unavailable in the presence of a large CFO.

Based on the relationship between the CFO and the resultant power degradation at the output of PN code correlator, a nondata-aided and nonphase-based AFC method suitable for digital broadcasting is proposed in this paper. To accelerate the tracking speed, the momentum steepest descent method (MSDM) [19] is adopted for its fast convergence and the $S$ curve is reshaped by a CFO-dependent factor. The proposed method has the advantages of wide tracking range (equal to twice the symbol rate and independent of both the phase keying number and the spreading code length), short tracking time and low implementation complexity, which make it attractive for digital broadcasting services.

The rest of this paper is organized as follows: the system model is described in Section II; Section III formulates the AFC algorithm and introduces a simple but effective reshaping function of the $S$ curve; in Section IV, both the transient-state and the steady-state performances of the proposed method are presented; the issue of implementation is discussed in Section V; and finally Section VI concludes this paper. 


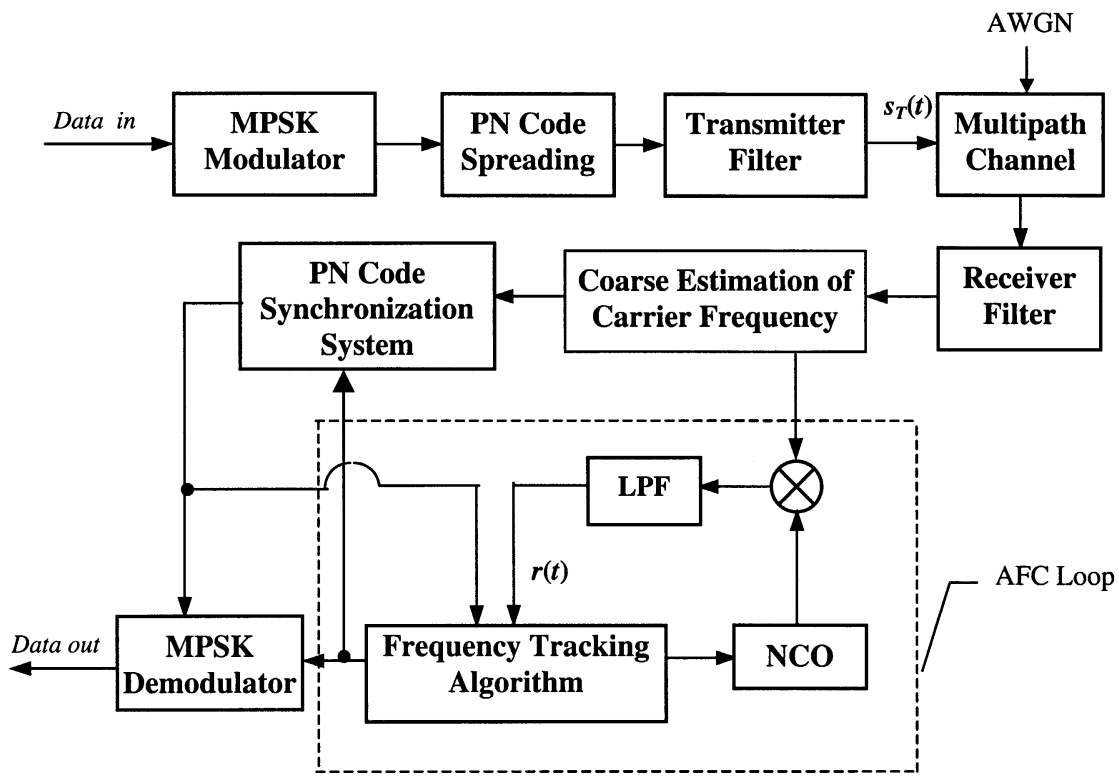

Fig. 1. System model of MPSK DS-CDMA transceiver with AFC loop.

\section{SYSTEM MODEL}

The system model of the MPSK DS/CDMA transceiver with AFC loop is depicted in Fig. 1. The input data is MPSK modulated and then spreaded by the PN code sequence $\left\{c_{n}\right\}$ of length $N$. The complex envelope of the modulated signal is given by

$$
s_{T}(t)=\sum_{l=-\infty}^{\infty} e^{j \phi_{l}} \sum_{n=0}^{N-1} c_{n} g_{T X}\left(t-n T_{c}-l T\right)
$$

where $T_{c}$ is the chip duration of $\left\{c_{n}\right\}, T$ is the symbol duration, $g_{T X}(t)$ is the impulse response of the transmitter filter and $\left\{\phi_{l}\right\}$ denote the modulation data taken from the MPSK constellation, which are assumed to be independently and identically distributed. The baseband signal $s_{T}(t)$ is then up-converted to the radio frequency and transmitted over a multipath channel. In a typical digital broadcasting channel [1]-[3], the received baseband signal can be given by

$$
r(t)=\sum_{l_{p}=0}^{L_{p}-1} a_{l_{p}} s_{T}\left(t-\tau_{l_{p}}\right) e^{j 2 \pi f_{d}\left(t-\tau_{l_{p}}\right)}+n(t),
$$

where $L_{p}$ denotes the total number of multipath reflections, $a_{l_{p}}$ and $\tau_{l_{p}}$ are the complex attenuation factor and the time delay associated with path $l_{p}$, respectively, $f_{d}$ represents the CFO between the down-converted signal and the output of NCO, and $n(t)$ is the additive white Gaussian noise (AWGN) with zero mean and variance $2 \sigma_{0}^{2}$. Assuming the maximum delay spread of this channel is smaller than $T$ while the minimum delay difference between successive paths is greater than $T_{c}$, then the multipath interferences can be neglected for carefully designed PN codes [13], [29]. We also assume that the strongest path has been acquired by the receiver, and the magnitude and the phase shift of this path are given by $A$ and $\theta$, respectively. Generally, the receiver operates in the frequency acquisition mode prior to the tracking mode of AFC, which will produce a coarse estimation of the carrier frequency. Although this coarse estimate may still deviate from the true carrier frequency, its accuracy is sufficient to enable the code synchronization be accomplished and the CFO be pulled into the tracking range of the AFC loop. In particular, by employing two-dimensional search [20]-[22] or blind Capon algorithm [23], PN code synchronization can be achieved in the presence of a sizable CFO. Therefore, frequency tracking can be performed after de-spreading with the assumption that code synchronization has been accomplished already. For discussion convenience, the frequency mixer is represented by an ideal multiplier in Fig. 1. The low pass filter (LPF) following the mixer suppresses the sum frequency and passes the difference frequency only, and the CFO-shifted signal is then applied to the AFC loop to bring $f_{d}$ to zero.

\section{Formulation of the Proposed AFC Method}

In this section, we will derive the proposed AFC method based on the CFO-induced power degradation, and introduce a reshaping function to accelerate the tracking speed of its $S$ curve.

To begin with, let us examine the correlator output of the strongest path under the assumption that PN code synchronization has already been achieved. Sampling $r(t)$ at the chip rate $1 / T_{c}$ yields

$r_{v}=A e^{j\left(2 \pi v f_{d} T_{c}+\theta\right)} \sum_{l=-\infty}^{\infty} e^{j \phi_{l}} \sum_{n=0}^{N-1} c_{n} g\left(v T_{c}-n T_{c}-l T\right)+\tilde{w}_{v}$

where $A$ and $\theta$ are constants independent of the CFO, $\tilde{w}_{v}=$ $\left.n(t) \otimes g_{R X}(t)\right|_{t=v T_{c}}=\tilde{w}_{I, v}+j \tilde{w}_{Q, v}, g_{R X}(t)$, is the impulse response of the receiver filter, symbol $\otimes$ denotes convolution, and $g(t)$ represents the convolution of the impulse response of the transmitter filter with that of the receiver filter, which satisfies the Nyquist pulse-shaping criterion for zero inter-symbol-interference (ISI) [24]. It follows that $\tilde{w}_{I, v}$ and $\tilde{w}_{Q, v}$ are mutually 


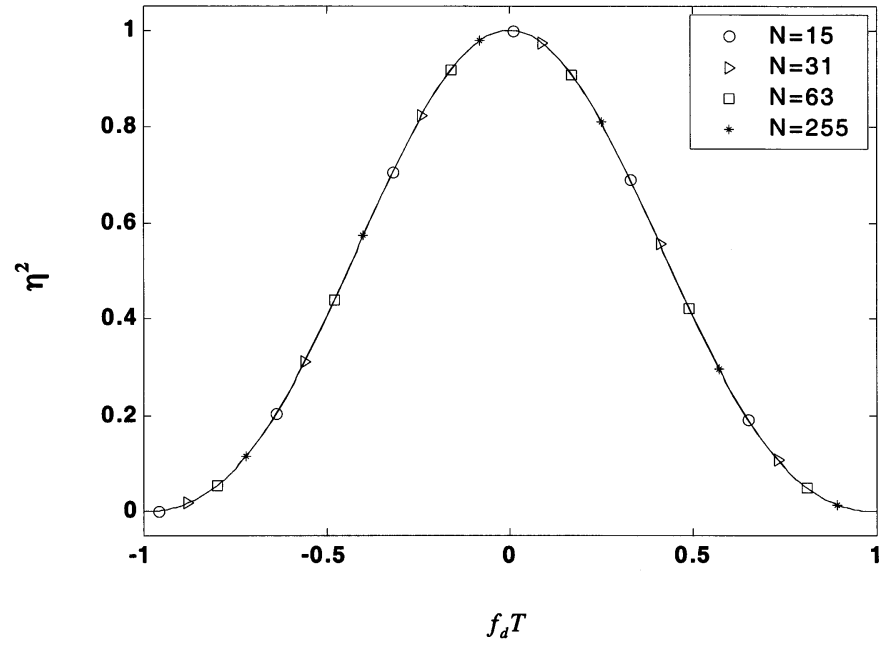

Fig. 2. The relationship between the power degradation factor $\eta^{2}$ and the normalized CFO $f_{d} T$, with $N$ as a parameter.

independent Gaussian noises with zero mean and variance $\sigma_{0}^{2}$. Then, $r_{v}$ is passed through the PN code correlator and the $k$-th correlator output is given by

$$
Z_{k}=\frac{1}{N} \sum_{v=k N}^{(k+1) N-1} r_{v} c_{v-k N}
$$

Without loss of generality, the term $A g(0) e^{j \theta}$ in (2) is normalized to unity. Let $w_{k}=(1 / N) \sum_{v=k N}^{(k+1) N-1} \tilde{w}_{v} c_{v-k N}, \tilde{\theta}=$ $\theta+\pi f_{d}\left(T-T_{c}\right)+\phi_{k}$ and

$$
\eta=\frac{\sin \left(\pi f_{d} T\right)}{N \sin \left(\pi f_{d} T_{c}\right)}
$$

(3) can be rewritten as

$$
Z_{k}=\eta \exp \left[j\left(\tilde{\theta}+2 \pi f_{d} k T\right)\right]+w_{k} .
$$

From (5), it is apparent that the presence of CFO $f_{d}$ will influence $Z_{k}$ in two different ways. Firstly, $f_{d}$ leads to the phase rotation of $Z_{k}$ (indicated by the exponential term), which has been utilized by phase-based algorithms [15]-[18] to build the CFO estimator in the form of $\arg \left(Z_{k}^{*} Z_{k+i}\right)$, where the superscript * denotes complex conjugate. Secondly, the magnitude of $Z_{k}$ is attenuated by a factor $\eta$, which is a function of $f_{d}$ as seen from (4). Based on this observation, we shall develop an AFC method based on the power degradation factor given by $\eta^{2}$. To the best knowledge of the authors, this approach has not been explored before.

Fig. 2 shows $\eta^{2}$ versus $f_{d} T$, the normalized CFO, for the range of $(-1,1)$, with $N$ as a parameter. It is clear from this figure that $\eta^{2}$ is a convex function of CFO for $f_{d} \in(-1 / T, 1 / T)$. Moreover, $\eta^{2}$ is almost independent of $N$ (the four curves overlap with each other) and reaches its maximum at $f_{d}=0$, which means there is no power loss if the NCO output frequency equals the carrier frequency of $r(t)$. Since the objective of AFC is to bring the CFO to zero, maximizing $\eta^{2}$ will eventually lead to $\eta^{2}=1$ and $f_{d}=0$, the desired result, provided $\left|f_{d}\right|<1 / T$. Taking $\psi=\left|Z_{k}\right|^{2}$ as the cost function of $f_{d}$, the task of frequency tracking can therefore be simplified into the recursive maximization of $\psi$ with respect to $f_{d}$. Various approaches including gradient algorithms can be applied here to build the recursive formula. In the current work the differential filtering steepest descent method (DFSDM) [19] is adopted, which is an improved steepest descent method since it employs better approximation to the gradient rather than the "simple difference" used by ordinary gradient methods. Consequently, DFSDM can accelerate the convergence without increasing the step size and it can also relax the bound on the step size. For simplicity, momentum filter, the simplest type of differential filter, is employed in the iteration formula given by

$$
f_{d, k+1}=(\rho+1) f_{d, k}-\rho f_{d, k-1}+\mu \nabla_{k}, \quad 0<|\rho|<1
$$

where $\rho$ is a constant, $\mu$ is the step size and $\nabla_{k}=$ $\partial\left(\left|Z_{k}\right|^{2}\right) / \partial f_{d, k}$ is the gradient. Substituting (2) into (3) yields

$\nabla_{k}=\frac{1}{N^{2}} \operatorname{Im}\left[\sum_{v=k N}^{(k+1) N-1} r_{v} c_{v-k N} \cdot \sum_{v=k N}^{(k+1) N-1} r_{v}^{*}(v-k N) c_{v-k N}\right]$, (7) where $\operatorname{Im}($.$) denotes the imaginary part of the inner variable.$ Let $W_{k}=(1 / N) \sum_{v=k N}^{(k+1) N-1} r_{v}(v-k N) c_{v-k N}$, (7) can be expressed by

$$
\nabla_{k}=\operatorname{Im}\left(Z_{k} W_{k}^{*}\right)
$$

The $S$ curveis a key characteristic curve that determines the tracking performance of AFC [25]-[27]. In the absence of timing error, the $S$ curve corresponding to (6) can be expressed by

$$
\begin{aligned}
S\left(f_{d}\right) & =\left.E\left[\nabla_{k}\right]\right|_{f_{d, k}=f_{d}} \\
& =-\frac{1}{N^{2}} \operatorname{Im}\left[\frac{1-e^{j 2 \pi f_{d} T}}{1-e^{j 2 \pi f_{d} T_{c}}} \sum_{n=0}^{N-1} n e^{-j 2 \pi f_{d} T_{c}}\right] .
\end{aligned}
$$

From (9), we obtain $f_{d} S\left(f_{d}\right)<0$ when $f_{d} \in(-1 / T, 1 / T)$ and $S\left(f_{d}\right)=0$ at $f_{d}= \pm 1 / T$. Therefore, the tracking range of (9) is given by $(-1 / T, 1 / T)$. We normalize (9) by its maximum magnitude and plot it in Fig. 3 for $f_{d} \in[-1 / T, 1 / T]$. Without considering the tracking range, we find that $S\left(f_{d}\right)$ is similar to the $S$ curves obtained by previous methods [15]-[18]. Since the restoring force of the AFC is proportional to the magnitude of its $S$ curve, a drawback of $S\left(f_{d}\right)$ lies in its nonuniformly-distributed magnitude. As a result, when $f_{d}$ goes beyond the frequency where $\left|S\left(f_{d}\right)\right|$ reaches its maximum, the rate of convergence will slow down due to the decreasing restoring force of AFC, which often occurs when the AFC first takes over from the acquisition mode.

To circumvent this shortcoming and obtain an AFC loop with more uniform restoring force, we propose to modify the $S$ curveby multiplying $S\left(f_{d}\right)$ with a reshaping function $\kappa\left(f_{d}\right)$, that is

$$
\bar{S}\left(f_{d}\right)=\kappa\left(f_{d}\right) S\left(f_{d}\right)
$$

To maintain the correct polarity of $S\left(f_{d}\right)$, the condition $\kappa\left(f_{d}\right)>$ 0 for $\left|f_{d}\right|<1 / T$ should be satisfied. Obviously, the choice of 


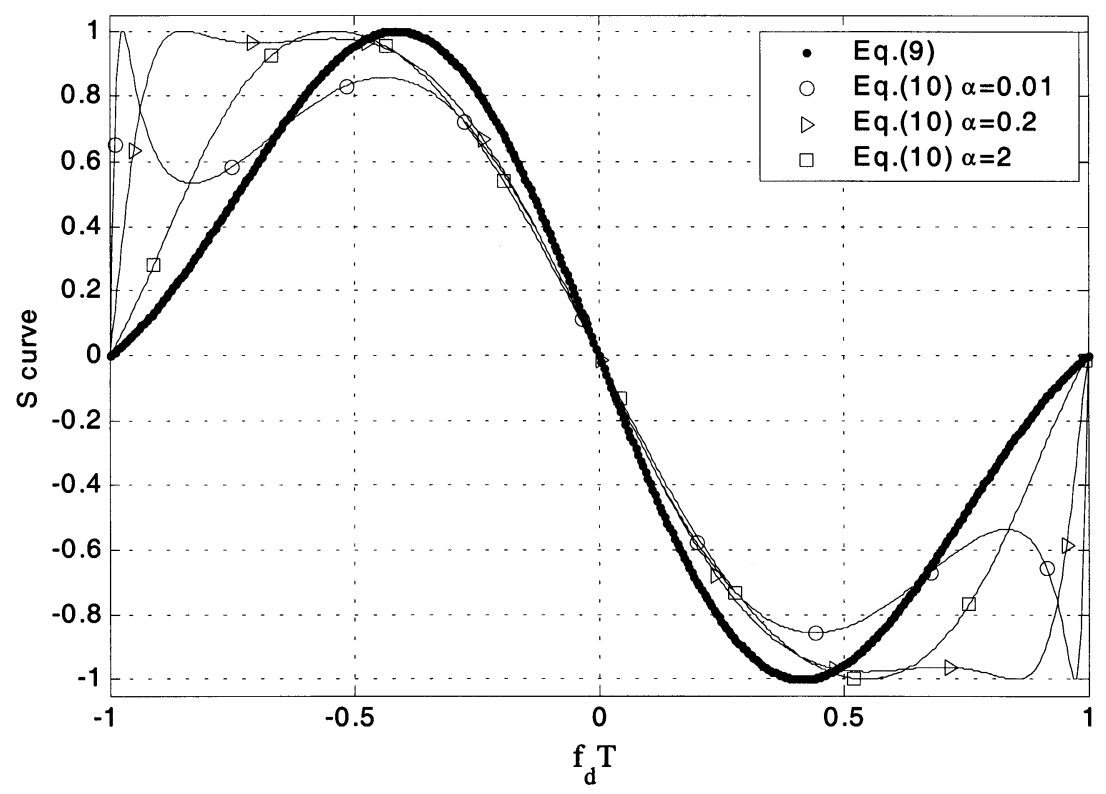

Fig. 3. Original $S$ curve $S\left(f_{d}\right)$ and modified $S$ curve $\bar{S}\left(f_{d}\right)$ versus normalized CFO $f_{d} T$. Different parameters $\alpha=0.01,0.2$ and 2 are employed in the reshaping function $\kappa\left(f_{d}\right)$.

$\kappa\left(f_{d}\right)$ is not unique. A simple but effective $\kappa\left(f_{d}\right)$ takes the form of

$$
\kappa\left(f_{d}\right)=\beta+\left.\frac{\gamma}{\operatorname{Re}\left(Z_{k} W_{k}^{*}\right)+\alpha}\right|_{f_{d, k}=f_{d}},
$$

where $\alpha, \beta$ and $\gamma$ are positive constants that are chosen to make the shape of $\bar{S}\left(f_{d}\right)$ approximate to that of the square wave, and $\operatorname{Re}\left(\right.$.) denotes the real part of the inner variable. Since $Z_{k}$ and $W_{k}$ are determined by $f_{d}$ only, in the sequel the index $k$ is omitted from $Z_{k}$ and $W_{k}$ wherever appropriate for notational convenience. In order to normalize the maximum magnitude of $\bar{S}\left(f_{d}\right)$ to unity, $\beta$ and $\gamma$ can be determined by $\beta=\left(\lambda_{1} \lambda_{3}\right)^{-1}$ and $\gamma=\left(\lambda_{2} \lambda_{3}\right)^{-1}$, where $\lambda_{1}=\max _{\left|f_{d}\right|<1 / T}\left[S\left(f_{d}\right)\right]$, $\lambda_{2}=\max _{\left|f_{d}\right|<1 / T}\left\{\left(S\left(f_{d}\right)\right) /\left(\operatorname{Re}\left(E\left[Z W^{*}\right]\right)+\alpha\right)\right\}$, $\lambda_{3}=\max _{\left|f_{d}\right|<1 / T}\left\{\left((S(f d)) / \underline{\lambda}_{1}\right)+\left(1 / \underline{\lambda}_{2}\right)\right.$. $\left.\left(\left(S\left(f_{d}\right)\right) /\left(\operatorname{Re}\left(E\left[Z W^{*}\right]\right)+\alpha\right)\right)\right\}$, and the symbol $\mathrm{E}[$. denotes expectation. With such definitions of $\beta$ and $\gamma$, the characteristics of $\bar{S}\left(f_{d}\right)$ is determined by $\alpha$. If $\alpha$ is chosen too large, there is little improvement for the shape $\bar{S}\left(f_{d}\right)$ in comparison with that of $S\left(f_{d}\right)$. On the other hand, if $\alpha$ is too small, the shape of $\bar{S}\left(f_{d}\right)$ fluctuates rapidly, which will lead to the undesirable frequency jitter. Since (11) is a nonlinear function of $f_{d}$, theoretical determination of suitable values for $\alpha$ is difficult. As an alternative, computer simulation is conducted, which indicates appropriate choices of $\alpha$ should belong to the range of $(0.15,0.25)$. To illustrate the impact of $\alpha$ on the modified $S$ curve, $\bar{S}\left(f_{d}\right)$ is plotted in Fig. 3 for $\alpha=0.01,0.2$ and 2. The choice of $\alpha=0.2$ is appropriate since it yields a close to ideal modified $S$ curve (square wave), whereas $\alpha=0.01$ and 2 are inappropriate choices, which may lead to the poor shape of $\bar{S}\left(f_{d}\right)$.

Inserting (11) into (6) finally yields the AFC algorithm proposed in this paper:

$$
f_{d, k+1}=(\rho+1) f_{d, k}-\rho f_{d, k-1}+\mu \kappa\left(f_{d, k}\right) \nabla_{k} .
$$

As a final remark of this section, we note that $\kappa\left(f_{d}\right)$ decreases monotonically over the range of $f_{d} \in(-1 / T, 1 / T)$. Combining $\kappa\left(f_{d}\right)$ with $\mu$, we can regard $\mu \kappa\left(f_{d}\right)$ as a variable step size that is able to dynamically control the convergence rate of the AFC in accordance with the size of CFO.

\section{Performance}

\section{A. Tracking Range}

To find the tracking range of the proposed method, we substitute (11) into (10) and obtain

$$
\bar{S}\left(f_{d}\right)=-\frac{\kappa\left(f_{d}\right)}{N^{2}} \operatorname{Im}\left[\frac{1-e^{j 2 \pi f_{d} T}}{1-e^{j 2 \pi f_{d} T_{c}}} \sum_{n=0}^{N-1} n e^{-j 2 \pi f_{d} T_{c}}\right] .
$$

From (13), the first pair of polar zero-crossing points are located at $f_{d}= \pm 1 / T$. Consequently, the tracking range of the proposed method is given by $(-1 / T, 1 / T)$, which is equivalent to twice the symbol rate and is independent of both the phase keying number $M$ and the spreading code length $N$. This feature makes the proposed method advantageous over previously presented AFC schemes [15]-[18], whose tracking range is inversely proportional to $M$.

\section{B. Transient-State Tracking Behavior}

To illustrate the efficacy of the reshaped $S$ curve, simulations are carried out to compare the transient tracking behavior of (6) and (12), and $\alpha=0.2$ is employed in the reshaping function of (11).

Let $S N R=\left(2 \sigma_{0}^{2}\right)^{-1}$, transient-state tracking behavior under $S N R=-5$ and $10 \mathrm{~dB}$ are plotted in Fig. 4(a) and Fig. 4(b), respectively. The system parameters used in this experiment are as follows: $1 / T=5 \times 10^{6}$ symbols/second, $f_{d, 0}=-0.99 / T$, $M=16, \mu=0.01$ and $N=255$. These trajectories indicate that (12) settles to the steady state much faster than (6). To 

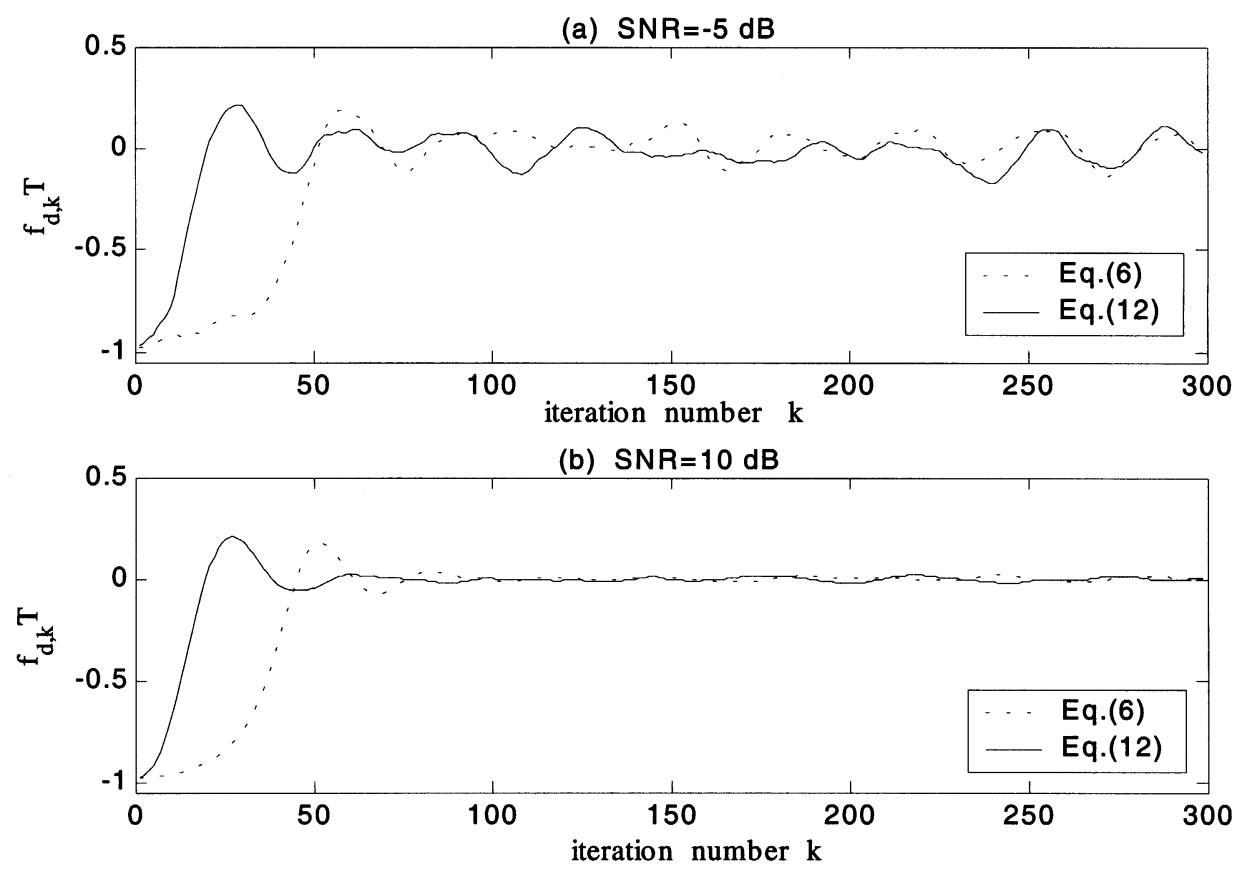

Fig. 4. Transient frequency tracking behavior resulted from algorithm (6) and (12).

TABLE I

Average Tracking Time of Algorithm

(6) AND (12) FOR DIFFERENT SYSTEM PARAMETERS AND SNR LEVEL

\begin{tabular}{ccccc}
\hline \hline & \multicolumn{4}{c}{$\bar{T}_{f}\left(M=16, N=255,1 / T=5 \times 10^{6}\right.$ symbols $\left./ \mathrm{s}\right)$} \\
\cline { 2 - 5 } $\begin{array}{c}\text { SNR } \\
(\mathrm{dB})\end{array}$ & \multicolumn{3}{c}{ Case 1} & \multicolumn{2}{c}{ Case 2 } \\
& \multicolumn{1}{c}{$\mu=0.01, \rho=0.88, f_{u, 0} T=-0.99$} & $\mu=05, \rho=0.90, f_{d, 0} T=0.99$ \\
\hline \hline-5 & 183 & 88 & 130 & 62 \\
0 & 157 & 74 & 105 & 41 \\
5 & 139 & 66 & 79 & 35 \\
10 & 122 & 59 & 65 & 28 \\
\hline \hline
\end{tabular}

quantify the tracking speed of AFC, we introduce a variable $T_{f}$, which satisfies $\left|f_{d, T_{f}}\right| \leq 0.01$. Due to the influence of noise, $T_{f}$ is a random variable. For this reason, we conduct $10^{3}$ simulation runs to acquire the average value of $T_{f}$, say $\bar{T}_{f}$. Table I summarizes the results of $\bar{T}_{f}$ obtained by (6) and (12) from two different experiments. An inspection of this table reveals that by employing (12) rather than $(6), \bar{T}_{f}$ can be significantly reduced. The efficacy of the modified $S$ curve is thereby demonstrated.

\section{Steady-State Tracking Behavior}

In order to obtain the analytical results concerning the steady-state tracking behavior, $\nabla_{k}$ is split into a deterministic part $S\left(f_{d, k}\right)$ and a random part $\hat{w}_{k}$, and (12) can thus be rewritten as

$$
f_{d, k+1}=(1+\rho) f_{d, k}-\rho f_{d, k-1}+\mu \bar{S}\left(f_{d, k}\right)+\mu \kappa\left(f_{d, k}\right) \hat{w}_{k},
$$

where

$$
\begin{gathered}
\hat{w}_{k}=-\frac{1}{N^{2}} \sum_{n=0}^{N-1} \sum_{m=0}^{N-1}-\frac{1}{N} \sum_{n=0}^{N-1} \tilde{w}_{Q, n}\left(W_{I, k}-n R_{I, k}\right) \\
+\frac{1}{N} \sum_{m=0}^{N-1} \tilde{w}_{I, m}\left(W_{Q, k}-m R_{Q, k}\right) .
\end{gathered}
$$

As shown in (A.12), (A.16) and (A.17) of the Appendix, the first and second moments of $\hat{w}_{k}$ are given by

$$
E\left[\hat{w}_{k}\right]=0
$$

and

$$
\begin{gathered}
E\left[\hat{w}_{k} \hat{w}_{k+p}\right]=\left\{\frac{\sigma_{0}^{2}}{N^{4}} \sum_{n_{1}=0}^{N-1} \sum_{n_{2}=0}^{N-1} \sum_{n_{3}=0}^{N-1}\left(n_{2} n_{3}+n_{1}^{2}-2 n_{1} n_{2}\right)\right. \\
\left.\times \cos \left[2 \pi T_{c} f_{d, k}\left(n_{2}-n_{3}\right)\right]+\frac{\sigma_{0}^{4}\left(N^{2}-1\right)}{6 N^{2}}\right\} \delta(p),
\end{gathered}
$$

respectively, where $\delta(p)$ is the Kronecker delta function. When $p=0$ and $f_{d, k}$ is sufficiently small, (17) reduces to

$$
\operatorname{Lim}_{f_{d, k} \rightarrow 0} E\left[\hat{w}_{k}^{2}\right] \approx \frac{\left(N^{2}-1\right) \sigma_{0}^{2}}{12 N^{2}}\left(N+2 \sigma_{0}^{2}\right) .
$$

Since we are interested in the steady-state performance only, the assumption of $f_{d, k} \approx 0$ is well justified in obtaining (18). Furthermore, (14) can be approximated linearly by

$$
f_{d, k+1}=\left(1+\rho+\mu K_{A}\right) f_{d, k}-\rho f_{d, k-1}+\mu \kappa_{0} \hat{w}_{k},
$$

where $K_{A} \triangleq \operatorname{Lim}_{f_{d} \rightarrow 0}\left(\left(\partial \bar{S}\left(f_{d}\right)\right) / \partial f_{d}\right)$ and $\kappa_{0} \triangleq \kappa(0)$. It follows from (19) that $\hat{w}_{k}$ and $f_{d, k}$ can be viewed as the input and output of a linear system with the transfer function of

$$
Q\left(z^{-1}\right)=\frac{\mu \kappa_{0} z^{-1}}{1-\left(1+\rho+\mu K_{A}\right) z^{-1}+\rho z^{-2}} .
$$


Following [28], the analytical expression for the statistical characteristics of $f_{d}$ are given by

$$
E\left[f_{d}\right]=0,
$$

and

$$
E\left[f_{d, k} f_{d, k+p}\right]=E\left[\hat{w}_{k} \hat{w}_{k+p}\right] \Theta R_{Q Q}(p),
$$

where $\Theta$ represents discrete convolution,

$$
\begin{aligned}
R_{Q Q}(p)= & (\bar{A}+\bar{B}) r_{1}^{p} \varepsilon(p)+(\bar{B}+\bar{C}) r_{2}^{p} \varepsilon(p)+(\bar{A}+\bar{B}) r_{1}^{-p} \\
& \times \varepsilon(-p-1)+(\bar{B}+\bar{C}) r_{2}^{-p} \varepsilon(-p-1), \\
\varepsilon(p)= & \left\{\begin{array}{ll}
1 & p \geq 0 \\
0 & p<0
\end{array},\right.
\end{aligned}
$$

$r_{1}$ and $r_{2}$ denote the roots of equation $r^{2}-\left(1+\rho+K_{A}\right) r+\rho=0$ and

$$
\begin{aligned}
& \bar{A}=\frac{\kappa_{0}^{2} \underline{\mu}^{2} \underline{r}_{1}^{2}}{\left(r_{1}-r_{2}\right)^{2}\left(1-r_{1}^{2}\right)}, \\
& \bar{B}=\frac{\kappa_{0}^{2} \mu^{2} r_{1} r_{2}}{\left(r_{1}-r_{2}\right)^{2}\left(r_{1} r_{2}-1\right)}, \\
& \bar{C}=\frac{\kappa_{0}^{2} \mu^{2} r_{2}^{2}}{\left(r_{1}-r_{2}\right)^{2}\left(1-r_{2}^{2}\right)} .
\end{aligned}
$$

Particularly, the variance of $f_{d}$ is

$$
\sigma_{f_{d}}^{2}=\sigma_{\hat{w}}^{2} R_{Q Q}(0)=\sigma_{\hat{w}}^{2}(\bar{A}+2 \bar{B}+\bar{C}) .
$$

Equation (21) indicates the proposed method is unbiased. Furthermore, $\sigma_{f_{d} T}^{2}$, the normalized variance of frequency jitter [14], [15], can be obtained by multiplying (28) with $T^{2}$.

Different from the phase-based methods with feedforward structure, which operate on finite-length observations and produce estimation of $f_{d}$ after processing a block of samples, the proposed algorithm works in a continuous way and makes use of all the past observations. In order to conduct a fair comparison of $\sigma_{f_{d} T}^{2}$ between the proposed algorithm and the phase-based methods, the model of equivalent estimator [27] is employed, with which algorithm (12) is transformed into an equivalent feedforward estimator with a finite observation length $L_{E}$. In line with this model, we let $\hat{f}_{d}$ denote the nominal frequency jitter of the equivalent estimator, which is related to the noise component $\hat{w}_{k}$ by

$$
\hat{f}_{d}=\frac{\kappa_{0}}{L_{E} K_{A}} \sum_{k=1}^{L_{E}} \hat{w}_{k} .
$$

Let $\sigma_{\hat{f}_{d}}^{2}$ denote the variance of $\hat{f}_{d}$; by keeping the mean square estimation errors unchanged, i.e. $\sigma_{\hat{f}_{d}}^{2}=\sigma_{f_{d}}^{2}$, we have

$$
L_{E}=\frac{1}{R_{Q Q}(0)}\left[\frac{6}{\pi T_{c}\left(N^{2}-1\right)}\right]^{2} .
$$

In the following, parameter $L_{E}$ is used as the common observation length to compare the variance of different AFC algorithms. To give an example, (30) is employed to calculate $L_{E}$
TABLE II

EQUIVALENT OBSERVATION LENGTH LE AND THE CORRESPONDING SYSTEM PARAMETERS

\begin{tabular}{ccccc}
\hline \hline \multirow{2}{*}{$L_{E}$} & \multicolumn{4}{c}{ System Parameters Corresponding to $L_{E}(M=2)$} \\
\cline { 2 - 5 } & $N$ & $1 / T$ & $\mu$ & $\rho$ \\
\hline 50 & 511 & $5 \times 10^{6}$ & 0.098 & 0.908 \\
100 & 255 & $5 \times 10^{6}$ & 0.191 & 0.817 \\
\hline \hline
\end{tabular}

that corresponds to the two sets of system parameters listed in Table II. For the first case, $L_{E}=50$; while for the second case, $L_{E}=100$.

The steady-state performances of the AFC schemes previously used for a fully digital MPSK DS/CDMA receiver have been comprehensively studied by [15]. The frequency jitter variances of the proposed method and the schemes given in [15], including DAFF, NDAFF and NDAFB, are now compared over the same $L_{E}$. Since $L_{E}$ must be an integer for DAFF and NDAFF, both of which are based on finite-length observations in practical applications, the system parameters are adjusted to satisfy such a requirement. The results for $f_{d, 0} T=0.1, L_{E}=50$ and 100 are shown in Fig. 5(a) and (b), and the corresponding system parameters are listed in Table II. The solid line labeled as "Theory" indicates the theoretical derivation given by (28), while the circles in Fig. 5(a) and (b) represent the Monte Carlo simulation result of algorithm (12) for SNR $\in\{-5,-3,0,3,10,12,15,18,20\} \mathrm{dB}$. The number of Monte Carlo runs is $10^{3}$ and for each run of simulation, $10^{4}$ samples of $f_{d, k}$ are taken after the condition $\left|f_{d, k} T\right| \leq 10^{-3}$ is met. As a benchmark, the variances of NDAFB, NDAFF and DAFF schemes are also shown. It is evident from these figures that the results obtained by Monte Carlo simulations agree well with the theoretical derivation, and the nondecision-aided and nonphase-based AFC method proposed in this paper outperforms the previously used phase-based schemes throughout the SNR level from $-5 \mathrm{~dB}$ to $15 \mathrm{~dB}$. The simulated BER performances of the proposed AFC method and the three phase-based schemes are shown in Fig. 6 for $f_{d, 0} T=0.1$. The equivalent observation length used in this simulation is $\mathrm{L}_{\mathrm{E}}=50$. Obviously, the proposed method has lower BER than the other three schemes, which is attributed to its better tracking accuracy as shown by Fig. 5 .

\section{IMPLEMENTATION}

In the proposed algorithm, gradient $\nabla_{k}$ must be generated at each iteration. As shown in (8), $\nabla_{k}$ can be expressed as the imaginary part of the product of $Z_{k}$ and $W_{k}^{*}$. Noting

$$
W_{k}=\frac{1}{N} \sum_{v=k N}^{(k+1) N-1}(v-k N) c_{v-k N} r_{v},
$$

it follows that $W_{k}$ can be obtained in the same way as $Z_{k}$ by multiplying $\left\{r_{v}\right\}$ with the weighted PN code sequence $\left\{n c_{n}\right\}$. Hence, we introduce a weighted correlator, which bears similar structure as the PN code correlator but has a set of different 
(a) $\mathrm{L}_{\mathrm{E}}=50$

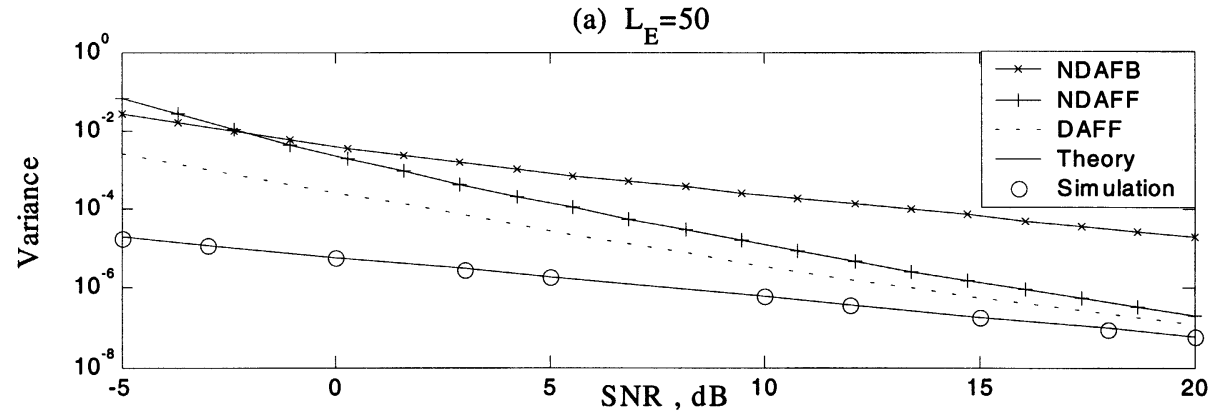

(b) $\mathrm{L}_{\mathrm{E}}=100$

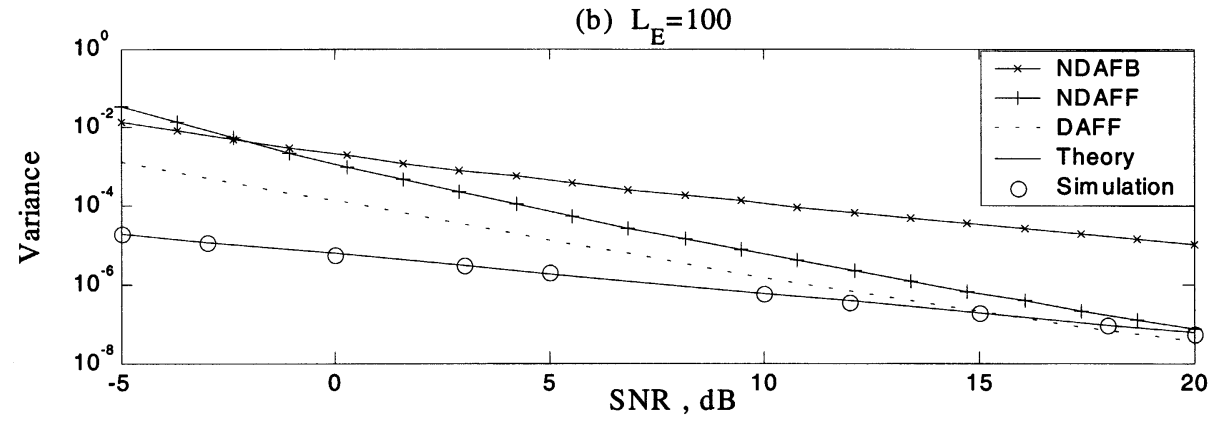

Fig. 5. Comparison of normalized variance of frequency jitter for $L_{E}=50$ and 100 .

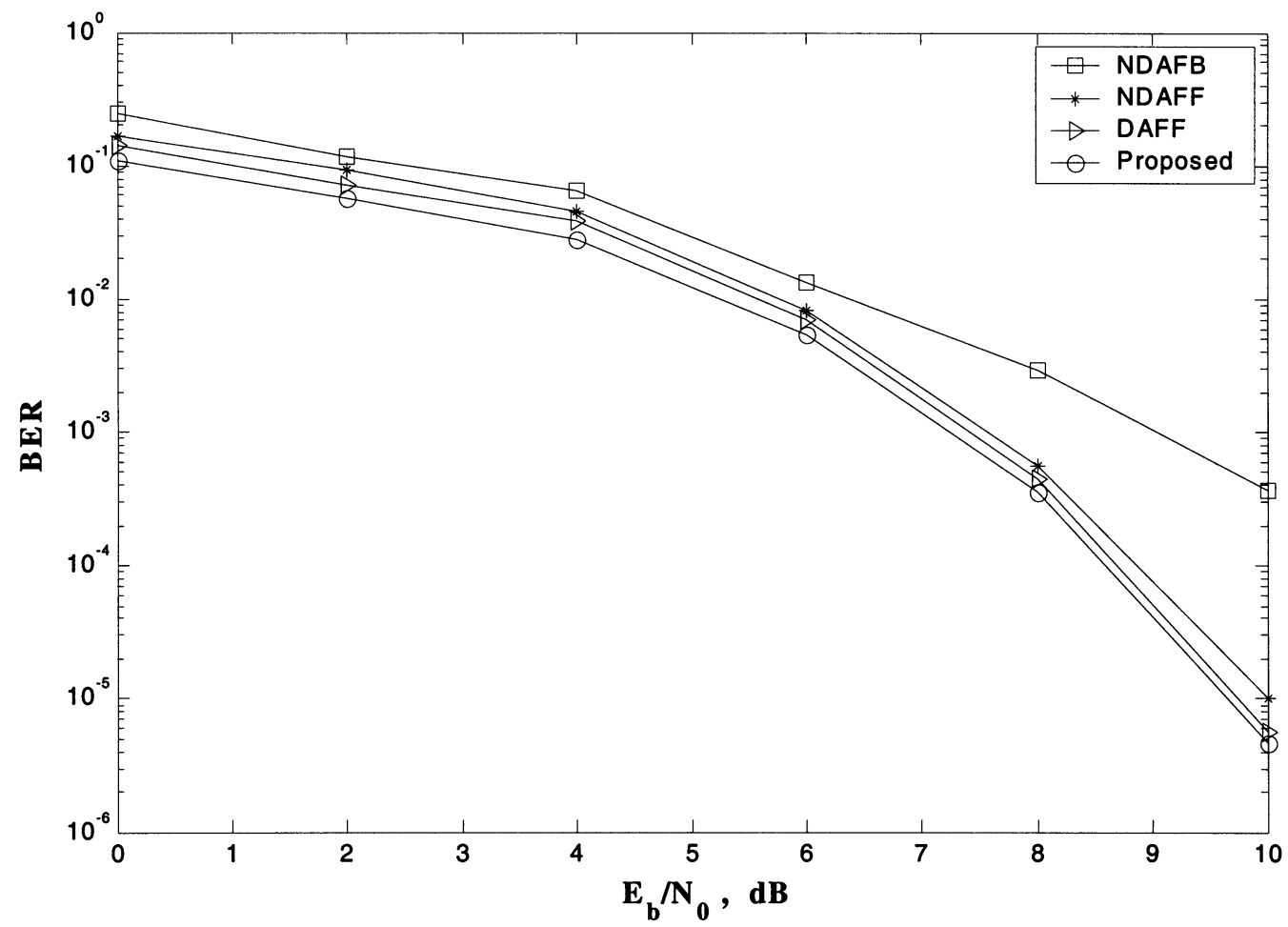

Fig. 6. Simulated BER performances of proposed AFC method, NDAFB, NDAFF, and DAFF schemes for $L_{E}=50$.

code sequence $\left\{\bar{c}_{n}\right\}$ given by $\bar{c}_{n}=n c_{n}$. As a result, $W_{k}$ can be realized through

$$
W_{k}=\frac{1}{N} \sum_{v=k N}^{(k+1) N-1} r_{v} \bar{c}_{v-k N}
$$

and the gradient $\nabla_{k}$ can be generated simply by means of correlation operations.
Fig. 7 gives the schematic diagram for the digital implementation of the proposed AFC method. Taking into consideration the effective tracking range of the algorithm, the bandwidth of the LPF should be no less than the symbol rate. The output of the LPF is sampled at the chip rate and the clock signal is furnished by the PN code synchronization system as shown by Fig. 1. Both the PN code correlator and the weighted correlator can be realized by means of standard $N$-tap FIR filters, whose tap weights are given by $\left\{c_{n}\right\}$ and $\left\{\bar{c}_{n}\right\}$, respectively. The output of 


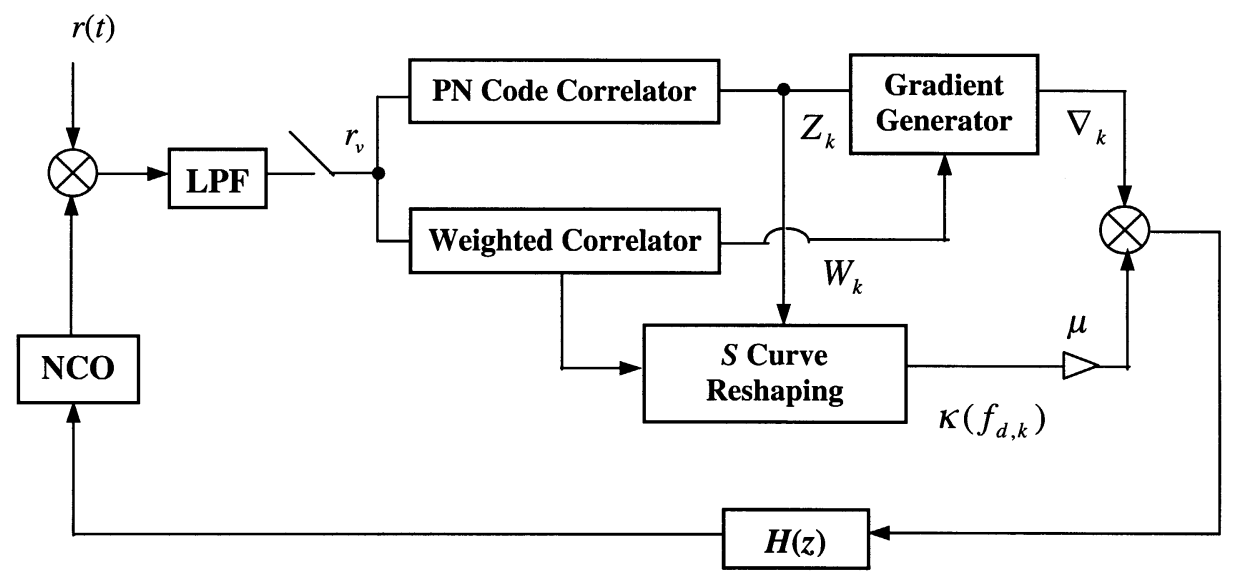

Fig. 7. Schematic diagram of digital implementation for the proposed frequency tracking method.

the FIR filters are sampled at symbol rate and the clock signal is also furnished by the PN code synchronization system. The gradient $\nabla_{k}$ is generated from the imaginary part of the product of the PN code correlator output and the complex conjugate of the weighted correlator output, as shown in (8) and (32). Furthermore, the real part of this product is used to compute the $S$ curve reshaping function $\kappa\left(f_{d, k}\right)$ as indicated in (11). Then, the gradient signal is multiplied by $\kappa\left(f_{d, k}\right)$ and $\mu$, and transformed into the control signal of NCO by the loop filter $H(z)$. In regard to the computation of $\kappa\left(f_{d, k}\right)$, once the parameter $\alpha$ is chosen, the other two coefficients $\beta$ and $\gamma$ are fixed. Therefore, only simple arithmetic operations are involved in computing $\kappa\left(f_{d, k}\right)$.

The computation load for each iteration of algorithm (12) requires a total of $(4 N+8)$ real products, 1 real division and $(4 N+2))$ real additions. It is worth noting that the intermediate variable $Z_{k}$ can also be used for subsequent data demodulation after the AFC loop settles to its steady state. Since PN code synchronization is necessary for DS/CDMA receivers, and the proposed AFC method can be realized by the correlation of code synchronization, its implementation complexity is very low. For digital broadcasting services based on MPSK DS/CDMA schemes, this feature is of great significance in reducing the implementation cost of frequency synchronization.

\section{CONCLUSION}

In this paper, a low-complexity AFC method which is nondecision-aided, nonphase-based and suitable for the fully digital MPSK DS/CDMA transceivers used in digital broadcasting has been proposed. Different from phase-based approaches, which recover carrier frequency from the CFO-induced phase rotation and suffer from the modulo- $2 \pi$ phase ambiguity, the proposed method utilizes the effect of power degradation at the correlator output and has an enlarged tracking range of twice the symbol rate, which is independent of both the phase keying number and the PN code length. To accelerate frequency tracking, an improved steepest descent algorithm has been adopted to build the iteration formula, and the $S$ curve has been modified by a simple but effective CFO-dependent reshaping function. Performance of the proposed method has been analyzed and compared with that of previously proposed phase-based schemes.

\section{APPENDIX}

In this appendix, key steps to compute $E\left[\hat{w}_{k}\right]$ and $E\left[\hat{w}_{k} \hat{w}_{k+p}\right]$ are outlined, where $\hat{w}_{k}$ is the random part of the gradient $\nabla_{k}$. Let $\hat{R}_{I, k}=\operatorname{Re}\left(Z_{k}\right), \hat{R}_{Q, k}=\operatorname{IM}\left(Z_{k}\right), \hat{G}_{I, k}=\operatorname{Re}\left(W_{k}\right), \hat{G}_{Q_{2} k}=$ $\operatorname{Im}\left(W_{k}\right), R_{I, k}=E\left[\hat{R}_{I, k}\right], R_{Q, k}=E\left[\hat{R}_{Q, k}\right], G_{I, k}=E\left[\hat{G}_{I, k}\right]$ and $G_{Q, k}=E\left[G_{Q, k}\right]$, then $\hat{w}_{k}$ is given by

$$
\begin{aligned}
\hat{w}_{k} \triangleq & \nabla_{k}-E\left[\nabla_{k}\right] \\
= & \left(\hat{R}_{I, k} \hat{G}_{Q, k}-\hat{R}_{Q, k} \hat{G}_{I, k}\right) \\
& -\left(R_{I, k} G_{Q, k}-R_{Q, k} G_{I, k}\right) .
\end{aligned}
$$

Substituting (3) and (8) into (A.1), the product of $\hat{w}_{k}$ and $\hat{w}_{k+p}$ can be represented by

$$
\hat{w}_{k} \hat{w}_{k+p}=\sum_{i=1}^{9} M_{i},
$$

where

$$
\begin{aligned}
M_{1}= & \frac{1}{N^{4}} \sum_{n_{1}=0}^{N-1} \sum_{n_{2}=0}^{N-1} \sum_{n_{3}=0}^{N-1} \sum_{n_{4}=0}^{N-1} \tilde{w}_{I, n_{1}} \tilde{w}_{I, n_{2}} \tilde{w}_{Q, n_{3}} \tilde{w}_{Q, n_{4}} \\
& \times\left(n_{1}-n_{3}\right)\left(n_{2}-n_{4}\right) \\
M_{2}= & \frac{1}{N^{3}} \sum_{n_{1}=0}^{N-1} \sum_{n_{2}=0}^{N-1} \sum_{n_{3}=0}^{N-1} \tilde{w}_{I, n_{1}} \tilde{w}_{Q, n_{2}} \tilde{w}_{Q, n_{3}} \\
& \times\left(n_{1}-n_{2}\right)\left(W_{I, k+p}-n_{3} R_{I, k+p}\right) \\
M_{3}= & -\frac{1}{N^{3}} \sum_{n_{1}=0}^{N-1} \sum_{n_{2}=0}^{N-1} \sum_{n_{3}=0}^{N-1} \tilde{w}_{I, n_{1}} \tilde{w}_{I, n_{2}} \tilde{w}_{Q, n_{3}} \\
& \times\left(n_{1}-n_{3}\right)\left(W_{Q, k+p}-n_{2} R_{Q, k+p}\right) \\
M_{4}= & \frac{1}{N^{3}} \sum_{n_{1}=0}^{N-1} \sum_{n_{2}=0}^{N-1} \sum_{n_{3}=0}^{N-1} \tilde{w}_{I, n_{1}} \tilde{w}_{Q, n_{2}} \tilde{w}_{Q, n_{3}} \\
& \times\left(n_{1}-n_{2}\right)\left(W_{I, k}-n_{3} R_{I, k}\right) \\
M_{5}= & \frac{1}{N^{2}} \sum_{n_{1}=0}^{N-1} \sum_{n_{2}=0}^{N-1} \tilde{w}_{Q, n_{1}} \tilde{w}_{Q, n_{2}} \\
& \times\left(W_{I, k}-n_{1} R_{I, k}\right)\left(W_{I, k+p}-n_{2} R_{I, k+p}\right) \\
M_{6}= & -\frac{1}{N^{2}} \sum_{n_{1}=0}^{N-1} \sum_{n_{2}=0}^{N-1} \tilde{w}_{Q, n_{1}} \tilde{w}_{I, n_{2}}
\end{aligned}
$$




$$
\begin{aligned}
& \times\left(W_{I, k}-n_{1} R_{I, k}\right)\left(W_{Q, k+p}-n_{2} R_{Q, k+p}\right), \\
M_{7}= & -\frac{1}{N^{3}} \sum_{n_{1}=0}^{N-1} \sum_{n_{2}=0}^{N-1} \sum_{n_{3}=0}^{N-1} \tilde{w}_{I, n_{1}} \tilde{w}_{I, n_{2}} \tilde{w}_{Q, n_{3}} \\
& \times\left(n_{1}-n_{3}\right)\left(W_{Q, k}-n_{2} R_{Q, k}\right), \\
M_{8}= & -\frac{1}{N^{2}} \sum_{n_{1}=0}^{N-1} \sum_{n_{2}=0}^{N-1} \tilde{w}_{I, n_{1}} \tilde{w}_{Q, n_{2}} \\
& \times\left(W_{Q, k}-n_{1} R_{Q, k}\right)\left(W_{I, k+p}-n_{2} R_{I, k+p}\right), \\
M_{9}= & \frac{1}{N^{2}} \sum_{n_{1}=0}^{N-1} \sum_{n_{2}=0}^{N-1} \tilde{w}_{I, n_{1}} \tilde{w}_{I, n_{2}} \\
& \times\left(W_{Q, k}-n_{1} R_{Q, k}\right)\left(W_{Q, k+p}-n_{2} R_{Q, k+p}\right) .
\end{aligned}
$$

As $\left\{\tilde{w}_{I, m}\right\}$ and $\left\{\tilde{w}_{Q, n}\right\}$ are zero mean and mutually independent, substituting $E\left[\tilde{w}_{I, m}\right]=E\left[\tilde{w}_{Q, n}\right]=E\left[\tilde{w}_{I, m} \tilde{w}_{Q, n}\right]=0$ and $E\left[\tilde{w}_{I, m} \tilde{w}_{I, n}\right]=E\left[\tilde{w}_{Q, m} \tilde{w}_{Q, n}\right]=\sigma_{0}^{2} \delta(m-n)$ into (A.1) and (A.3)-(A.11) leads to:

$$
\begin{aligned}
E\left[\hat{w}_{k}\right]= & 0 \\
E\left[M_{1}\right]= & \frac{\sigma_{0}\left(N^{2}-1\right)}{6 N^{2}} \delta(p), \\
E\left[M_{5}+M_{9}\right]= & \frac{\sigma_{0}^{2}}{N^{4}} \sum_{n_{1}=0}^{N-1} \sum_{n_{2}=0}^{N-1} \sum_{n_{3}=0}^{N-1}\left(n_{2} n_{3}+n_{1}^{2}-2 n_{1} n_{2}\right) \\
& \times \cos \left[2 \pi T_{c} f_{d, k}\left(n_{2}-n_{3}\right)\right] \delta(p) \\
E\left[M_{2}\right]= & E\left[M_{3}\right]=E\left[M_{4}\right]=E\left[M_{6}\right] \\
= & E\left[M_{7}\right]=E\left[M_{8}\right]=0 .
\end{aligned}
$$

Substituting (A.13)-(A.15) into (A.2) and combining all of the nonzero terms yields

$$
\begin{gathered}
E\left[\hat{w}_{k} \hat{w}_{k+p}\right]=\left\{\frac{\sigma_{0}^{2}}{N^{4}} \sum_{n_{1}=0}^{N-1} \sum_{n_{2}=0}^{N-1} \sum_{n_{3}=0}^{N-1}\left(n_{2} n_{3}+n_{1}^{2}-2 n_{1} n_{2}\right)\right. \\
\left.\times \cos \left[2 \pi T f_{d, k}\left(n_{2}-n_{3}\right)\right]+\frac{\sigma_{0}^{4}\left(N^{2}-1\right)}{6 N^{2}}\right\} \delta(p) .
\end{gathered}
$$

When $p=0$, (A.16) reduces to

$$
E\left[\hat{w}_{k}^{2}\right]=\frac{\left(N^{2}-1\right) \sigma_{0}^{2}}{12 N^{2}}\left(N+2 \sigma_{0}^{2}\right) .
$$

For $N \gg 1$,

$$
E\left[\hat{w}_{k}^{2}\right] \approx \frac{\sigma_{0}^{2}\left(N+2 \sigma_{0}^{2}\right)}{12}
$$

\section{REFERENCES}

[1] E. Geraniotis and B. Ghaffari, "Broadcast capability of direct sequence and hybrid spread spectrum," IEEE. J. Select. Areas Commun., vol. 8, pp. 489-502, May 1990.

[2] G. Carrere, "Comparison between CDMA and COFDM transmission techniques for the digital audio broadcasting by satellite," in Proc. 3rd European Conference on Satellite Communications, Nov. 1993, pp. $44-49$.
[3] A. Franchi and C. Eila, "Estimation of multipath channels for satellite digital broadcasting using synchronous CDMA," in Proc. IEEE ISSSTA'94, Jul. 1994, pp. 642-646.

[4] L. P. Clare, J. E. Baker, and A. R. K. Sastry, "A new CDMA scheme for multicasting using information classes," in Proc. IEEE Milcom'94, Oct. 1994, pp. 962-966.

[5] S. Rakib, "An innovative cable modem system for broadband communication," in Proc. International Broadcasting Convention, Sept. 1997, pp. $24-30$.

[6] J. G. Zhang, "Novel optical fiber code-division multiple access networks supporting real-time multichannel VBR distributions," IEEE Trans. Broadcast., vol. 43, pp. 339-349, Sept. 1997.

[7] M. Y. Liu and H. W. Tsao, "Real-time multimedia broadcasting via direct-detection fiber-optic synchronous CDMA networks," IEEE Trans. Broadcast., vol. 44, pp. 371-380, Sept. 1998.

[8] J. G. Zhang, "Flexible optical fiber CDMA networks using strict optical orthogonal codes for multimedia broadcasting and distribution applications," IEEE Trans. Broadcast., vol. 45, pp. 106-115, Mar. 1999.

[9] M. Chrysochoos and J. Kim, "Performance analysis of an MC-CDMA broadcasting system under high power amplifier nonlinearies: system proposal," IEEE Trans. Broadcast., vol. 46, pp. 256-262, Dec. 2000.

[10] P. Prasad and J. Farserotu, "ATM over Ka-band SATCOM via CDMA," in Proc. IEEE Globecom'98, Nov. 1998, pp. 1858-1864.

[11] L. Dongwook and L. B. Milstein, "Comparison of multicarrier DS-CDMA broadcast systems in a multipath fading channel," IEEE Trans. Commun., vol. 47, pp. 1897-1904, Dec. 1999.

[12] A. Stok and E. H. Sargent, "System performance comparison of optical CDMA and WDMA in a broadcast local area network," IEEE Commun. Lett., pp. 409-411, Sept. 2002.

[13] J. Viterbi, CDMA: Principles of Spread Spectrum Communication: Addison-Wesley Co., 1995.

[14] J. Farserotu and R. Prasad, IP/ATM Mobile Satellite Networks: Artech House Publishers, 2002.

[15] O. C. Mauss, F. Classen, and H. Meyr, "Carrier frequency recovery for a fully digital direct-sequence spread-spectrum receiver: a comparison," in Proc. VTC'93, 1993, pp. 392-395.

[16] F. D. Natali, "AFC tracking algorithms," IEEE Trans. Commun., vol. 32, no. 8, pp. 935-947, Aug. 1984.

[17] M. Luise and R. Reggiannini, "Carrier frequency recovery in all-digital modems for burst-mode transmissions," IEEE Trans. Commun., vol. 43, pp. 1169-1178, Apr. 1995

[18] U. Mengali and M. Morelli, "Data-aided frequency estimation for burst digital transmission," IEEE Trans. Commun., vol. 45, pp. 23-25, Jan. 1997.

[19] V. Solo and X. Kong, Adaptive Signal Processing Algorithms. Englewood Cliffs, New Jersey: Prentice Hall, Inc., 1995.

[20] S. L. Su, N. Y. Yen, and S. C. Hsieh, "Code acquisition of direct-sequence spread spectrum communication with Doppler shift," in Proc. IEEE ICC'95, vol. 3, pp. 1663-1667.

[21] U. Cheng, W. J. Herd, and J. I. Statman, "Spread-spectrum code acquisition in the presence of Doppler shift and data modulation," IEEE Trans. Commun, vol. 38, pp. 241-250, Feb. 1990.

[22] R. D. Gaudenzi, F. Giannetti, and M. Luise, "Signal synchronization for direct-sequence code-division multiple access radio modems," $E u$ ropean Trans. Telecom., vol. 9, pp. 73-89, Jan/Feb. 1998.

[23] H. B. Li and R. H. Wang, "A novel blind code synchronization scheme for DS-CDMA systems in multipath fading channels," in Proc. IEEE Globecom'01, vol. 2, pp. 1395-1399.

[24] G. L. Stuber, Principles of Mobile Communication, 2nd ed: Kluwer Academic Publishers, 2001

[25] J. Lei and T. S. Ng, "New AFC algorithm for a fully-digial DS/CDMA receiver," in Proc. IEEE ISCAS'01, vol. 4, 2001, pp. 294-297.

[26] H. Meyr and G. Ascheid, Synchronization in Digital Communications. New York: John Wiley \& Sons, 1990, vol. 1.

[27] U. Mengali and A. N. D'Andrea, Synchronization Techniques for Digital Receivers, 1990.

[28] V. Oppenheim and R. W. Schafer, Discrete-Time Signal Processing. Englewood Cliffs, New Jersey: Prentice Hall Inc., 1989.

[29] G. L. Turin, "Introduction to spread-spectrum anti-multipath techniques and their application to urban digital radio," Proc. IEEE, vol. 68, pp. 328-353, Mar. 1980. 